\title{
Angle-dependent reflection, transmission and absorption coefficients measurement using a 2D waveguide
}

\author{
Ze Zhang ${ }^{\mathrm{a}, \mathrm{c}, *}$, Hervé Denayer ${ }^{\mathrm{a}, \mathrm{c}}$, Claus Claeys ${ }^{\mathrm{a}, \mathrm{c}}$, Wim Desmet ${ }^{\mathrm{a}, \mathrm{c}}$, Elke Deckers ${ }^{\mathrm{b}, \mathrm{c}}$ \\ ${ }^{a}$ KU Leuven, Dept. of Mechanical Engineering, Celestijnenlaan 300B, 3001 Leuven, Belgium \\ ${ }^{b}$ KU Leuven, Campus Diepenbeek, Dept. of Mechanical Engineering, Wetenschapspark 28, 3590 \\ Diepenbeek, Belgium \\ ${ }^{c}$ DMMS lab, Flanders Make, Belgium
}

\begin{abstract}
This paper presents a way to obtain angle-dependent reflection, transmission and absorption coefficients of a sample based on the modal decomposition method in a rectangular waveguide. Microphone locations are optimized to ensure that the modal decomposition can be applied. The method is subsequently validated both numerically and experimentally.
\end{abstract}

Keywords: Modal decomposition, Angle-dependent absorption coefficient, Angle-dependent transmission coefficient, Material characterization method

\section{Introduction}

Impedance tubes have been used extensively to characterize the acoustic impedance, reflection, transmission and absorption coefficients of a material. It is based on the 1D plane wave propagation assumption, which implies that these properties can only be 5 determined in the context of normal incidence[1]. In case that the sample is isotropic, the frequency-dependent characteristic impedance and wavenumber can be calculated by measuring the reflection and transmission coefficients at normal incidence with anechoic terminations both up- and down-stream. These two parameters can in turn be used to calculate the angle-dependent reflection and transmission coefficients of the sample using the transfer matrix method [2]. This is not the case for non-isotropic

${ }^{*}$ Corresponding author, E-mail address: ze.zhang@kuleuven.be. 
materials, such as artificially designed metamaterials [3]. Thus, it is desirable to devise a way to measure the angle-dependent coefficients in this case directly. Preferably, these angle-dependent properties should correspond to the idealized case, i.e., the sample is of infinite extent and no spurious reflections occur.

Various efforts have been made to tackle this issue. One approach uses the so-called in-situ measurements based on the free-field assumption [4-7], which usually involves a sound source and one or multiple receivers at the same side of the sample. By processing the signals measured by the receivers, the reflected field can be separated from the total field and the angle-dependent absorption coefficient can be obtained. However, there are various drawbacks. Firstly, to ensure the accuracy of the measurement, the sample has to be considerably large even under normal incidence conditions to avoid the edge diffraction. This requirement on the sample size becomes even more stringent under an oblique incidence. For example, paper [6] reports that to characterize the absorption coefficient of a melamine foam slab starting at $750 \mathrm{~Hz}$ and an angle of incidence of 60 degrees, the size of the sample has to be $1.8 \mathrm{~m}$ by $1.2 \mathrm{~m}$ (as compared to $0.264 \mathrm{~m}$ by $0.045 \mathrm{~m}$ using the method adopted in this paper), which might not be realistic at an early stage of the product development. Secondly, very accurate positioning of the sound source and receivers is required to make the measurement less susceptible to noise. Thirdly, as this approach was first proposed by the building acoustics community, the incentive to measure the sound field on the other side of the sample is lacking, as a result, the transmission coefficient cannot be readily measured.

Another free-field approach uses the spatial Fourier-transform approach as proposed by Tamura et al. [8]. It assumes any pressure field can be characterized as a finite sum of plane wave fields of different directions. By measuring at a sufficient number of locations at various planes parallel to the sample surface, the amplitude of each plane wave can be solved. However, as this is still a free-field method, it falls a victim to all the drawbacks mentioned in the previous paragraph. Prisutova et al. [9] used a square duct to quantify the reflection and absorption coefficients of each mode based on the axial wave numbers using the spatial Fourier-transform approach. In that

40 setup, the locations of the sound sources are constant and the duct is $4.15 \mathrm{~m}$ long to allow the microphones moving axially to obtain a sufficient number of measurements. 
Additionally, the microphones are intrusive since the pressure at multiple locations of each cross-section needs to be measured. Secondly, there are no clear metrics for choosing the sound source and microphone locations, which potentially poses an issue 45 of signal to noise ratio (SNR) when considering a larger number of modes. As a result, only the most dominant mode at a frequency range can be quantified in the paper. Thirdly, the amplitude of each mode is not calculated directly from the measurement but optimized to match with the measurement, which makes it hard to guarantee that this method will yield consistent characterization outcomes. Lastly, the relationship between the angle-dependent absorption coefficient and absorption coefficient of each mode is not explicitly established.

The modal decomposition approach has been proved to be capable of characterizing a sample beyond the plane wave regime[10-14]. One advantage of this method over the spatial Fourier-transform method is its non-intrusiveness since it is sufficient to ${ }_{55}$ measure the pressure just at the boundaries to fully determine the system. Schultz et al. [15] proposed to use the modal decomposition approach in a square duct to extend the frequency range of an impedance tube measurement. Though one can argue that the same setup can be used to characterize the angle-dependent absorption coefficient of a sample, there are several considerations. Firstly, the relationship between the angledependent absorption coefficient and that of each mode is not proposed in the paper since it aims to address another issue. Secondly, the locations of the microphones are not chosen in a systematic way and this important choice is not straightforward to make in case where a large number of modes have to be accounted for. Thirdly, in that paper, transmission coefficient characterization is not conducted due to the setup used.

In this paper, we adopt the modal decomposition method to quantify the angledependent reflection coefficient $(R)$, transmission coefficient $(T)$ and absorption coefficient $(\alpha)$ of a sample in a rectangular waveguide. The microphone locations are optimized over the frequency range of interest to ensure that the method can be applied and the system is less liable to noise. Based on the measurement, the scattering matrix 70 of the system is calculated and is subsequently used to obtain the angle-dependent coefficients, considering the proposed relationship between the angle-dependent absorption coefficient and that of each mode. Both numerical and experimental validations show 
a good agreement with the prediction.

This paper is organized as follows: section 2 describes the methodology used to obtain the angle-dependent coefficients, in section 3 , the selection of the measurement locations based on the optimization approach is proposed, section 4 outlines the numerical and experiment setup to validate the method, section 5 presents the results from both simulation and experiment and the conclusions are summarized in section 6 based on the results.

\section{Theoretical background}

In this part, firstly, the modal decomposition theory is discussed. Moreover, based on the theory, the methodology used to calculate the angle-dependent coefficients is presented. Finally, an optimization scheme to ensure that the above-mentioned methodology can be applied in real-life is presented.

\subsection{Acoustic propagation in a rectangular waveguide}

In a homogeneous medium, the propagation of acoustic waves is governed by the Helmholtz equation, which takes the form:

$$
\nabla^{2} P(x, y, z)+k^{2} P(x, y, z)=0,
$$

where $P(x, y, z)$ is the location-dependent pressure with the time term eliminated, $\nabla^{2}$ is the Laplace operator and $k$ is the acoustic wavenumber, which is defined as:

$$
k=\frac{2 \pi f}{c}=\frac{\omega}{c},
$$

where $\omega$ is the angular frequency, $f$ is the frequency of interest and $c$ is the sound speed in the medium. For a waveguide with a rectangular cross-section and rigid boundaries as shown in figure 1 if the direction of propagation is the $z$-direction, the solution of equation (1) which is expressed as a finite sum of contributions from each duct mode takes the form [16]:

$$
P(x, y, z)=\sum_{m, n}\left(P_{m, n}^{+} \Psi_{m, n}^{+}(x, y) e^{-j k_{m, n}^{+} z}+P_{m, n}^{-} \Psi_{m, n}^{-}(x, y) e^{j k_{m, n}^{-} z}\right),
$$


where $x, y, z$ are defined according to the coordinate system in figure 11, superscripts

${ }^{+}$and $^{-}$denote positive and negative direction of propagation, subscripts ${ }_{m, n}$ refers to mode $(m, n)$ with $m$ in the $x$-direction and $n$ in the $y$-direction, $P_{m, n}^{ \pm}$is the pressure amplitude of mode $(m, n), k_{m, n}^{ \pm}$is the wavenumber for mode $(m, n), \Psi_{m, n}^{ \pm}(x, y)$ is the pressure pattern on the cross-section for mode $(m, n)$, which is defined as [16]:

$$
\Psi_{m, n}^{ \pm}(x, y)=C_{m, n} \cos \left(k_{x} x\right) \cos \left(k_{y} y\right)
$$

where $k_{x}$ and $k_{y}$ are the wavenumber on $x$ - and $y$-direction respectively, being defined as:

$$
k_{x}=\frac{m \pi}{W}, \quad k_{y}=\frac{n \pi}{H},
$$

where $W$ and $H$ are dimensions of the waveguide in the $x$ - and $y$-direction in figure 1. $C_{m, n}$ is the scaling factor which is introduced to normalize the acoustic intensity of different duct modes, which is defined as:

$$
C_{m, n}=\sqrt{\left(2-\delta_{m, 0}\right)\left(2-\delta_{n, 0}\right)},
$$

where $\delta_{i, j}$ is the Kronecker delta. The wavenumber of each mode in the $z$-direction

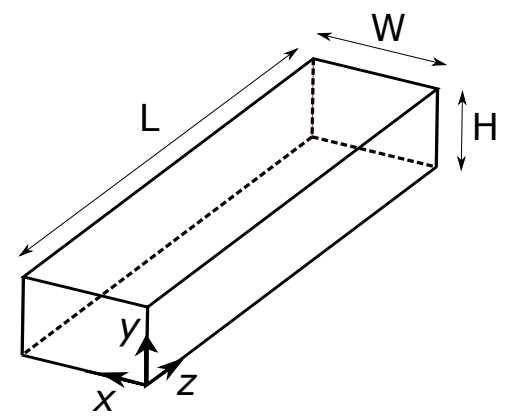

Figure 1: Definition of the coordinate system and the dimensions for a waveguide with a rectangular crosssection

$k_{m, n}^{ \pm}$is given by:

$$
k_{m, n}^{ \pm}=\sqrt{k^{2}-\left(k_{x}\right)^{2}-\left(k_{y}\right)^{2}} .
$$

For a combination of $m$ and $n$ that yields a real $k_{m, n}^{ \pm}$, the mode $(m, n)$ is called to be cut-on, which means that the waves corresponding to this mode can propagate along 
the $z$-direction; otherwise it is a cut-off mode, which means it is evanescent and cannot propagate along the $z$-direction. \\ 2.2. Modal characterization realization}

This part extends the previously presented equations to the matrix form, in order that the modal matrix, scattering matrix and angle-dependent coefficients can be calculated based on the measurements. Moreover, the considerations to determine the dimensions of the waveguide are presented.

\section{${ }_{95}$ 2.2.1. Calculation of the model matrix}

The acoustic field in a waveguide with rigid boundaries can be expressed as an finite sum of cut-on modes assuming that the contributions of cut-off (evanescent) modes are negligible at locations far away from the source, obstacles or discontinuities. Moreover, if $H$ is sufficiently small to only enable the $0^{\text {th }}$ order propagating mode, all quantities are considered to be independent from $y$. As a result, the matrix form of equation (3) considering $N$ modes in the $x$-direction takes the form:

$$
P(x, z) \approx\left[\begin{array}{c}
\Psi_{0,0}^{+}(x) e^{-j k_{0,0}^{+} z} \\
\vdots \\
\Psi_{N-1,0}^{+}(x) e^{-j k_{N-1,0}^{+} z} \\
\Psi_{0,0}^{-}(x) e^{+j k_{0,0}^{-} z} \\
\vdots \\
\Psi_{N-1,0}^{-}(x) e^{+j k_{N-1,0}^{-} z}
\end{array}\right]^{\top}\left[\begin{array}{c}
P_{0,0}^{+} \\
\vdots \\
P_{N-1,0}^{+} \\
P_{0,0}^{-} \\
\vdots \\
P_{N-1,0}^{-}
\end{array}\right] .
$$

When considering $s$ microphone locations, equation $(8)$ can be further expanded to:

$$
\left[\begin{array}{c}
P\left(x_{1}, z_{1}\right) \\
\vdots \\
P\left(x_{s}, z_{s}\right)
\end{array}\right]=\underline{M} \vec{P},
$$


where modal matrix $\underline{M}$ is given by:

$$
\underline{M}=\left[\begin{array}{ccc}
\Psi_{0,0}^{+}\left(x_{1}\right) e^{-j k_{0,0}^{+} z_{1}} & \ldots & \Psi_{0,0}^{+}\left(x_{s}\right) e^{-j k_{0,0}^{+} z_{s}} \\
\vdots & \vdots & \vdots \\
\Psi_{N-1,0}^{+}\left(x_{1}\right) e^{-j k_{N-1,0}^{+} z_{1}} & \ldots & \Psi_{N-1,0}^{+}\left(x_{s}\right) e^{-j k_{N-1,0}^{+} z_{s}} \\
\Psi_{0,0}^{-}\left(x_{1}\right) e^{+j k_{0,0}^{-} z_{1}} & \ldots & \Psi_{0,0}^{-}\left(x_{s}\right) e^{+j k_{0,0}^{-} z_{s}} \\
\vdots & \vdots & \vdots \\
\Psi_{N-1,0}^{-}\left(x_{1}\right) e^{+j k_{N-1,0}^{-} z_{1}} & \ldots & \Psi_{N-1,0}^{-}\left(x_{S}\right) e^{+j k_{N-1,0}^{-} z_{s}}
\end{array}\right]^{\top}
$$

and the vector of pressure amplitude of each mode $\vec{P}$ is:

$$
\vec{P}=\left[\begin{array}{c}
P_{0,0}^{+} \\
\vdots \\
P_{N-1,0}^{+} \\
P_{0,0}^{-} \\
\vdots \\
P_{N-1,0}^{-}
\end{array}\right]
$$

As a result, $\vec{P}$ can be calculated by [17]:

$$
\vec{P}=\underline{M^{+}}\left[\begin{array}{c}
P\left(x_{1}, z_{1}\right) \\
\vdots \\
P\left(x_{s}, z_{s}\right)
\end{array}\right],
$$

where $\underline{M}^{+}$denotes the Moore-Penrose pseudo-inverse of matrix $\underline{M}$. It is worth noting that for a total number of $N$ cut-on modes in the $x$-direction, $s$ cannot be smaller than $2 N$ for a $\underline{M}$ of full rank.

\subsubsection{Calculation of the scattering matrix}

To characterize the relationship between the output and the input of a system, a scattering matrix $\underline{S}$ is often used, which can be expressed as:

$$
\left[\begin{array}{c}
P_{o}^{+} \\
P_{i}^{-}
\end{array}\right]=\left[\begin{array}{ll}
\underline{T^{+}} & \underline{R^{-}} \\
\underline{R^{+}} & \underline{T^{-}}
\end{array}\right]\left[\begin{array}{c}
P_{i}^{+} \\
P_{o}^{-}
\end{array}\right]=\underline{S}\left[\begin{array}{c}
P_{i}^{+} \\
P_{o}^{-}
\end{array}\right],
$$


where $P_{i}^{ \pm}$and $P_{o}^{ \pm}$are the pressure amplitude of each mode in the positive and negative $z$-direction, as shown in figure $2, \underline{R^{ \pm}}$and $\underline{T^{ \pm}}$are matrices of reflection and transmission coefficients of different modes with positive and negative $z$-direction incidences.

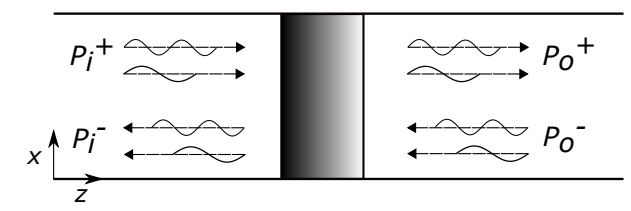

Figure 2: A schematic of $\underline{S}$ calculation model

Considering $N$ cut-on modes, the size of $\underline{S}$ is $2 N \times 2 N$. Given that during each measurement a total number of $s=2 N$ independent points are measured, thus at least $2 N$ independent measurements are needed to obtain $\underline{S}$. In our implementation, the independent measurements are achieved by varying the location of the sound source, i.e., by using the multiple source method [14]. Based on this reasoning, the scattering matrix $\underline{S}$ can be calculated as:

$$
\underline{S}=\left[\begin{array}{ccc}
P_{o, 0,1}^{+} & \ldots & P_{o, 0,2 N}^{+} \\
\vdots & & \vdots \\
P_{o, N-1,1}^{+} & & P_{o, N-1,2 N}^{+} \\
P_{i, 0,1}^{-} & & P_{i, 0,2 N}^{-} \\
\vdots & & \vdots \\
P_{i, N-1,1}^{-} & \ldots & P_{i, N-1,2 N}^{-}
\end{array}\right]\left[\begin{array}{ccc}
P_{i, 0,1}^{+} & \cdots & P_{i, 0,2 N}^{+} \\
\vdots & & \vdots \\
P_{i, N-1,1}^{+} & & P_{i, N-1,2 N}^{+} \\
P_{o, 0,1}^{-} & & P_{o, 0,2 N}^{-} \\
\vdots & & \vdots \\
P_{o, N-1,1}^{-} & \ldots & P_{o, N-1,2 N}^{-}
\end{array}\right]^{-1}
$$

where in $P_{*, k, l}^{ \pm}, k$ denotes the $k^{\text {th }}$ mode, $l$ denotes the $l^{\text {th }}$ measurement. One can see that $\underline{S}$ is dependent on the inversion of $\underline{M}$ since $P_{*, k, l}^{ \pm}$is obtained from the inversion of $\underline{M}$. Hence the condition number of $\underline{M}$ needs to be small to ensure an accurate $\underline{S}$.

\subsubsection{Calculation of the absorption coefficient}

The aim is to measure the angle-dependent absorption coefficient $\alpha_{o}$ in an idealized way, i.e., the sample is infinitely wide under an oblique incidence. Besides, the environment is assumed to be anechoic which means no sound waves should be reflected back to the sample. 
The angle-dependent absorption coefficient in this case can be extracted from $S$ measured in a waveguide at an angle of incidence which corresponds to a mode. In case when $H$ is sufficiently small, according to equation (5), $k_{y}$ equals 0 . As a result, the axial wavenumber $k_{m, 0}^{ \pm}$can be calculated as:

$$
k_{m, 0}^{ \pm}=\sqrt{k^{2}-k_{x}^{2}}
$$

The angle $\theta$ as measured from the normal incidence can be calculated as:

$$
\theta_{m, 0}=\operatorname{atan}\left(\frac{k_{x}}{k_{m, 0}^{ \pm}}\right) .
$$

This equation relates an oblique angle of incidence $\theta_{m, 0}$ to a certain mode $m$. Thus, a plane wave incidence at a certain angle can be regarded as a mode with a wave vector corresponding to that angle. As a result, by combining equation (5), (15) and 16 together and ranging $m$ from the first to the last cut-on mode, an angle of incidence from 0 to 90 degrees can be discretely covered. The absorption coefficient $\alpha(\theta)_{m, 0}$ under oblique angle of incidence $\theta_{m, 0}$ can be obtained as the absorption coefficient of $m^{\text {th }}$ mode $\alpha_{m}$, which, in the idealized case, is defined as:

$$
\alpha(\theta)_{m, 0}=\alpha_{m}=1-\left|\underline{S}_{(1: N, m)}\right|^{2}-\left|\underline{S}_{((N+1): 2 N, m)}\right|^{2},
$$

where $\underline{S}_{(k, l)}$ denotes the element which is at $k^{\text {th }}$ row and $l^{\text {th }}$ column of $\underline{S}, \underline{S}_{(1: N, m)}$ corresponds to the transmission coefficients to various modes from the $m^{\text {th }}$ mode incidence and $\underline{S}_{((N+1): 2 N, m)}$ corresponds to the reflection coefficients from the $m^{\text {th }}$ mode. It is worth mentioning that, for a given mode, equation (17) incorporates both $0^{\text {th }}$ mode (specular) and higher order modes (diffraction) for both reflected and transmitted waves. One benefit of this implementation is for periodically arranged materials such as metamaterials, higher order diffractions which are induced by the periodicity can be substantial under certain circumstances and should not be ignored.

It is also worth noting that as the definition of the pressure pattern of each mode is symmetric, the absorption coefficient measured with mirrored angle of incidences will be the same, which is a limitation of the method under certain circumstances. 


\subsection{Determination of the dimensions of the waveguide}

As described in the previous section, there exists a relationship between the angledependent absorption coefficient and that of each cut-on mode. Consequently, a larger number of cut-on modes will lead to a finer set of discrete angles at which incidence can be calculated. The total number of cut-on modes $N$ (including the $0^{\text {th }}$ mode) on the $x$-direction of the rectangular waveguide as shown in figure 1 is decided by:

$$
N=\left\lceil\frac{2 f W}{c}\right\rceil,
$$

where $\lceil\cdot\rceil$ is the ceiling function. Thus, $W$ can be decided by the desired total number of cut-on modes $N$.

\section{3. Optimization of microphone locations}

In this section, a way to determine the microphone locations based on optimization is introduced, which robustifies the method against measurement noise. The initial pattern, objective function and optimization setup are described.

\subsection{The initial pattern}

From equation (12), 14] and (17), it is clear that $\alpha(\theta)_{m, 0}$ depends on the inversion of the modal matrix $\underline{M}$, which means that a modal matrix with a high condition number will yield a result which is prone to error. From equation (10) one may conclude that $\underline{M}$ depends only on the modal pressure pattern and the microphone locations at a given frequency. The acoustic modal pressure pattern are known once the dimensions of the cross-section are fixed. Thus, special attention should be given to the microphone locations to ensure an overall low condition number of $\underline{M}$.

Finding the global minimum is not set to be the goal. There are two reasons. First, a local minimum can also yield a workable microphone layout. Second, a local minimum can be obtained more efficiently using a gradient based solver. As this problem contains many local minima, the choice of the initial pattern is important.

A pattern with microphone locations evenly-spaced along two parallel lines parallel to the sample interface is selected. Firstly, it can be seen as a straightforward extension 
to the 1D impedance tube microphone pattern which should already give a reasonable result. Secondly, to show that this pattern performs better than a pattern of random points, the two condition number curves are compared in a duct of $0.8 \mathrm{~m}$ width. The reasoning for comparing to the random pattern is that the a random pattern presents a typical choice one would get without prior knowledge. Both patterns are depicted in figure 3 The spacing between the two lines of the two-line pattern is $0.03 \mathrm{~m}$. The reasoning is that the value approximates to a quarter of the wavelength at the maximum frequency $(3000 \mathrm{~Hz})$, so that all frequencies within the range should be possible to be characterized.

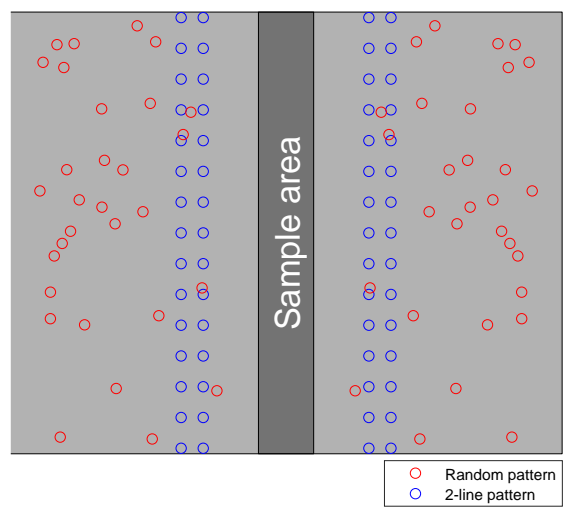

Figure 3: An illustration of the two-line pattern and a random pattern

As can be seen in figure 4 the condition number curve of the two-line case seems to perform overall better, especially in terms of peaks which do not coincide with cut-on frequencies and in the the range starting from $1600 \mathrm{~Hz}$ to $3000 \mathrm{~Hz}$.

For an impedance tube, the spacing between the two points can be varied to target different frequency regions. Similarly, the spacing between two lines for the two-line case can be adjusted to target a certain frequency range. To this end, the condition numbers of the pattern with different spacing is evaluated and shown in figure 5. It should be noted that the distance between the two lines and the sample surface is unspecified since it does not influence the condition number of the modal matrix. For a spacing increasing from $2 \mathrm{~cm}$ to $4 \mathrm{~cm}$, an overall decrease of the condition number is observed between $f_{1}=1000 \mathrm{~Hz}$ and $f_{2}=3000 \mathrm{~Hz}$. When the spacing increases further 


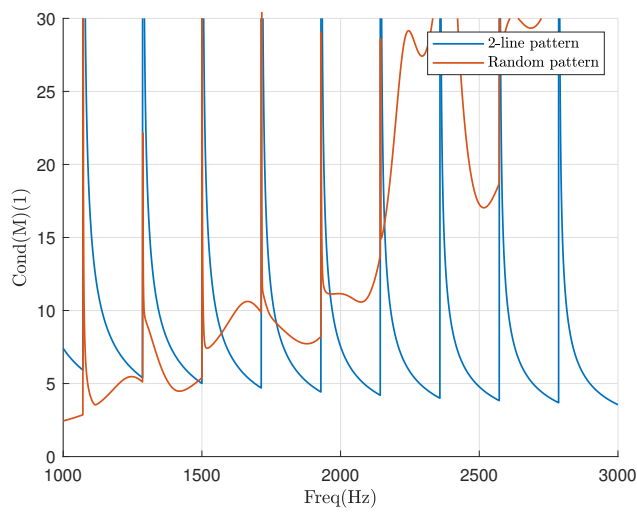

Figure 4: A typical condition number curve of two-line case with a $3 \mathrm{~cm}$ spacing and that of random points

from $4 \mathrm{~cm}$ to $7 \mathrm{~cm}$, the condition number continues to decrease at low frequencies, but a substantial increase can be observed at high frequencies. Looking at a wider frequency spectrum, the two observations can be unified as: the larger the spacing, the more favorable it is to the lower end of the spectrum, however, it comes at the cost of deteriorated matrix conditioning at higher frequencies. This can be explained by the fact that sound waves at a high frequency are characterized by a smaller wavelength, thus the microphones need to be closer to each other to capture these acoustic waves properly. It is also clear that the choice of the spacing is dependent on the frequency range that is of interest. Specifically, in the range of $1000 \mathrm{~Hz}$ to $3000 \mathrm{~Hz}$, a two-line pattern with a spacing of $4 \mathrm{~cm}$ maintains a fairly flat and generally low condition number throughout the spectrum.

\subsection{The objective function}

As mentioned in section 3.1 the condition number of $\underline{M}$ is a good metric to optimize. Thus, the condition number should be incorporated into the objective function. As the frequency increases, the number of cut-on modes increases accordingly. For a given number of microphones, the level of over-determination decreases with frequency. To this end, the weighted average of the condition number of $\underline{M}$ with a higher priority given to the high frequencies within the frequency range of interest is chosen as the objective function. The weighting is increased for each frequency band, which is separated by 

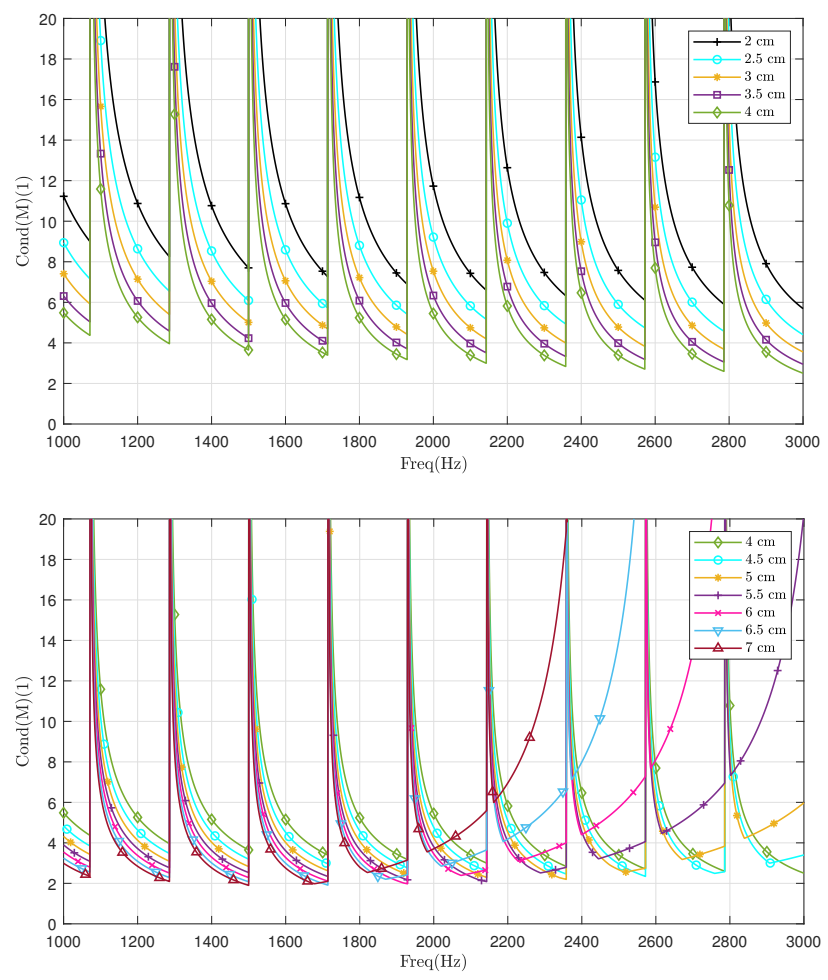

Figure 5: Condition number of two-line case with different spacings. Top - spacing from $2 \mathrm{~cm}$ to $4 \mathrm{~cm}$. Bottom - spacing from $4 \mathrm{~cm}$ to $7 \mathrm{~cm}$.

two cut-on frequencies. Since the modal matrix is singular at each cut-on frequency, the frequencies around each cut-on frequency are omitted in the evaluation. Concretely, the objective function $F\left(x_{m}, z_{m}, f\right)$ is defined as:

$$
F\left(x_{m}, z_{m}, f\right)=\frac{1}{N_{f b}} \sum_{i=1}^{N_{f} b} w_{i}(f) C\left(x_{m}, z_{m}, f\right)_{i} .
$$

In this equation, $N_{f b}$ is the number of frequency bands within the frequency range of interest, $i$ represents the $i^{\text {th }}$ frequency band within the range, $w_{i}$ is the weighting factor within the $i^{\text {th }}$ frequency band and $C\left(x_{m}, z_{m}, f\right)_{i}$ is the linear average of the condition number for location- and frequency-dependent modal matrix in the $i^{\text {th }}$ frequency band, evaluated at discrete frequencies within the band with a sufficiently fine resolution to ensure no relevant peaks are overlooked, and $x_{m}$ and $z_{m}$ are microphone locations. 
$C\left(x_{m}, z_{m}, f\right)_{i}$ is defined as:

$$
C\left(x_{m}, z_{m}, f\right)_{i}=\overline{\operatorname{cond}\left(\underline{M}\left(x_{m}, z_{m}, f\right)_{i}\right.},
$$

where cond is the operator to calculate the condition number at discrete frequency points. The weighting factor of each frequency band $w_{i}$ is determined based on the number of cut-on modes:

$$
w_{i}=\frac{1}{N_{2}-N_{i}+1},
$$

where $N_{2}$ is the number of cut-on modes at the maximum frequency of interest and $N_{i}$ represents the number of cut-on modes of the $i^{\text {th }}$ frequency band. As the condition number for an array with finite axial length will unavoidably be high at frequencies around a cut-on frequency and considering the condition number curve shape around each cut-on frequency (as shown in figure 5), the lower bound of each frequency band is set to be $10 \mathrm{~Hz}$ after the previous cut-on frequency and the upper bound is set to be $1 \mathrm{~Hz}$ before the next cut-on frequency.

\subsection{Optimization approach}

As mentioned earlier, the goal of the optimization is to define a microphone layout that exhibits a low condition number (average below 5) over the complete frequency range of interest, where singularities only occur around the cut-on frequencies, where a local minimum serves the need. The initial pattern is a two-line pattern with a $4 \mathrm{~cm}$ spacing in between, as described in section 3.1

Additionally, some constraints need to be accounted for. Equation (8) assumes the evanescent waves can be neglected at each microphone position. Hence, a distance should be kept between any microphone location and the inlet or outlet of the waveguide or the sample (the white regions of figure 65. Besides, a minimum distance between any two microphone locations should be set to allow the physical installation of the sensors in the setup. The optimization is performed in MATLAB using fmincon with a sequential quadratic programming (SQP) optimization algorithm which satisfies bounds

at all iterations [18]. As the modal matrix of the waveguide is sample-independent, the optimized pattern is optimal regardless of the sample type and sample thickness. 


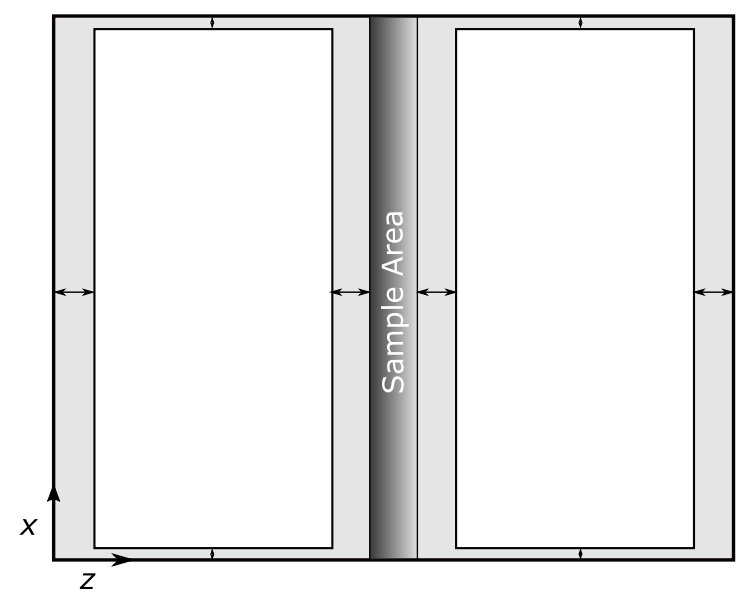

Figure 6: An illustration of the candidate region for the optimization

\section{Numerical and experimental setup}

This section introduces the setup for both the numerical models and experiments. It will also concretize the dimensions of the waveguide using the methodology described in section 2.3 .

\subsection{Sample characterization in a conventional impedance tube}

To validate that accurate $\alpha$ can be measured using the proposed methodology, an open-cell porous material is chosen and measured in an impedance tube using the two cavity method [19], which retrieves the equivalent characteristic impedance and wavenumber from two measurements with different backing cavity depths. Figure 7 shows the schematic representation of a measurement of a sample inside the impedance tube with a backing cavity.

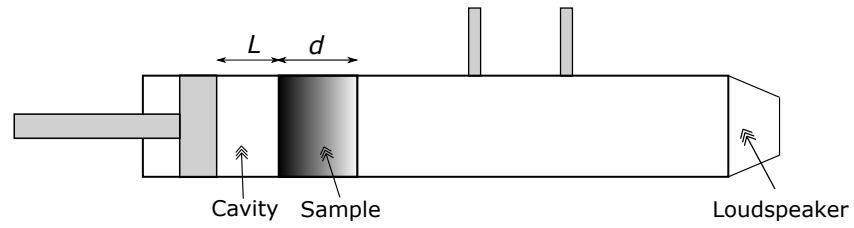

Figure 7: Schematic of an impedance tube measurement 
The frequency-dependent complex equivalent characteristic impedance $Z_{f}$ and wavenumber $k_{f}$ can be calculated as:

$$
\begin{gathered}
Z_{f}=\sqrt{\left(\frac{\left.Z_{0} Z_{0}^{\prime}\left(Z_{1}-Z_{1}^{\prime}\right)-Z_{1} Z_{1}^{\prime}\left(Z_{0}-Z_{0}^{\prime}\right)\right)}{\left(Z_{1}-Z_{1}^{\prime}\right)-\left(Z_{0}-Z_{0}^{\prime}\right)}\right),} \\
k_{f}=\frac{1}{2 j d} \ln \left(\frac{Z_{0}+Z_{c}}{Z_{0}-Z_{c}} \frac{Z_{1}+Z_{c}}{Z_{1}-Z_{c}}\right),
\end{gathered}
$$

where $d$ is the thickness of the sample used in the measurement, $Z_{0}$ and $Z_{0}^{\prime}$ are the surface impedance of the samples with two different backing air cavity depths and $Z_{1}$ and $Z_{1}^{\prime}$ represent the impedance at the back of the sample for the two different cavities. $Z_{0}$ and $Z_{0}^{\prime}$ are measured directly, $Z_{1}$ and $Z_{1}^{\prime}$ are calculated by:

$$
Z_{1}=-j Z \cot (k L)
$$

where $Z$ is the characteristic impedance of air and $L$ is the cavity depth. Finally, the absorption coefficient $\alpha$ in this case can be calculated by $Z_{1}$ and $Z_{a}$ :

$$
\alpha=1-\left(\frac{Z_{1}-Z}{Z_{1}+Z}\right)^{2}
$$

The measurement is carried out using the impedance tube at KU Leuven (figure 8) with a LMS Scadas Mobile at a bandwidth of $4096 \mathrm{~Hz}$. The number of averages of each run is 25 . The samples measured in the impedance tube are shown in figure 9 The thickness of each sample is $4 \mathrm{~cm}$. The two cavity depths are $2 \mathrm{~cm}$ and $6 \mathrm{~cm}$. They are chosen to avoid that the surface impedance from two measurements to be identical, at which the method is compromised. The current values ensure this error does not occur below $4280 \mathrm{~Hz}$.

The sample is also measured attached to the rigid backing, without a backing air layer for validation purpose. This measurement will be compared to a direct prediction of the absorption coefficient of a sample with a rigid backing without a backing air layer, using the material parameters obtained from the two previous measurements. In this case, the absorption coefficient can be obtained using the following equations:

$$
Z_{s 0}=-j Z_{s} \cot \left(k_{s} d\right)
$$




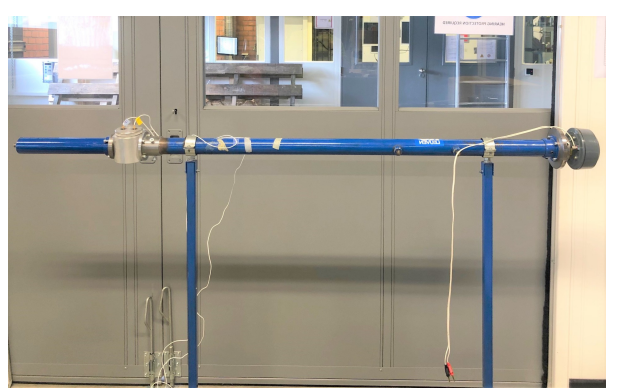

Figure 8: Impedance tube used for the measurement 20

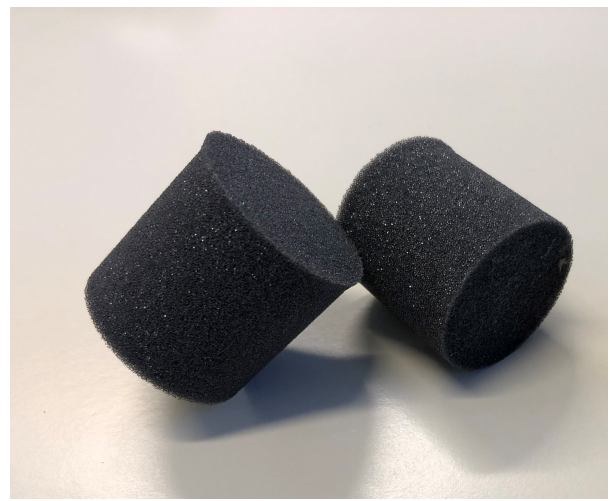

Figure 9: Samples used in the impedance measurement

$$
\begin{gathered}
R_{0}=\frac{Z_{s 0}-Z}{Z_{s 0}+Z}, \\
\alpha_{0}=1-\left|R_{0}\right|^{2},
\end{gathered}
$$

\subsection{Dimensions and microphone locations of the waveguide}

From equation (18), one may conclude that the wider the waveguide, the more cut-on modes will be present at a given frequency, thus the finer the resolution of the angledependent absorption coefficient. Connecting this with the practical consideration for manufacturing, the width of the waveguide ( $W$ in figure 1 ) is chosen as $0.8 \mathrm{~m}$, which allows for 9 cut-on modes at $2000 \mathrm{~Hz}$, i.e., the frequency of interest. At $3000 \mathrm{~Hz}$, which 
is the maximum frequency considered, it allows for 14 cut-on modes. The height of the waveguide cross-section ( $H$ in figure 1 ) is chosen as $4.5 \mathrm{~cm}$, which does not allow any higher mode propagation in that dimension until $3800 \mathrm{~Hz}$. For accuracy, microphone locations are bound to a rectangular region wherein a point is at least $5 \mathrm{~cm}$ away from the sample to avoid evanescent waves and the opening and is $1 \mathrm{~cm}$ away from two side boundaries of the waveguide. The $5 \mathrm{~cm}$ restriction is due to the observation that the distortion of the pressure field occurs mainly in that range. The minimum distance between any two microphones is set to $2 \mathrm{~cm}$, which is due to the size of microphone holder.

Based on the methodology presented in section 3.2 the objective function $F\left(x_{m}, z_{m}, f\right)$ (equation (19)) is optimized in the range from $1000 \mathrm{~Hz}$ to $3000 \mathrm{~Hz}$ considering a step of $1 \mathrm{~Hz}$. A total number of 30 microphone locations at each side of the sample is chosen to make the modal matrix overdetermined over the chosen frequency range based on the reasoning in section 2.2.1. The two-line pattern with a spacing of $4 \mathrm{~cm}$ of section 3.1 is chosen as the initial pattern for the gradient based optimization. After the optimization, the microphone locations are rounded to a precision of $5 \mathrm{~mm}$ to facilitate the manufacturing. Figure 10 shows the initial pattern, the optimized pattern and the optimized pattern where the microphone locations are rounded, together with their weighted condition number $F\left(x_{m}, z_{m}, f\right)$ (equation (19)). Figure 11 shows the condition number as a function of frequency for the three patterns, where $F\left(x_{m}, z_{m}, f\right)$ is clearly reduced after the optimization (from 1.41 to 0.76 ). Furthermore, the rounding of the microphone locations only slightly alters $F\left(x_{m}, z_{m}, f\right)$.

Due to the definition of the modal matrix, for a given pattern, any translational operation along the $z$-direction will not change the condition number of the modal matrix. Moreover, mirroring operations along either the central axial line of the duct or any line parallel to the sample surface do not change the condition number. These properties are used to define the microphone array layout for the right part of the waveguide in figure 10 , based on the optimized result for the left side. The optimized 255 pattern is shifted in $z$-direction to the opening by $8 \mathrm{~cm}$ in experiment to make it centered in the duct segment without changing the condition number based on the above reasoning. 


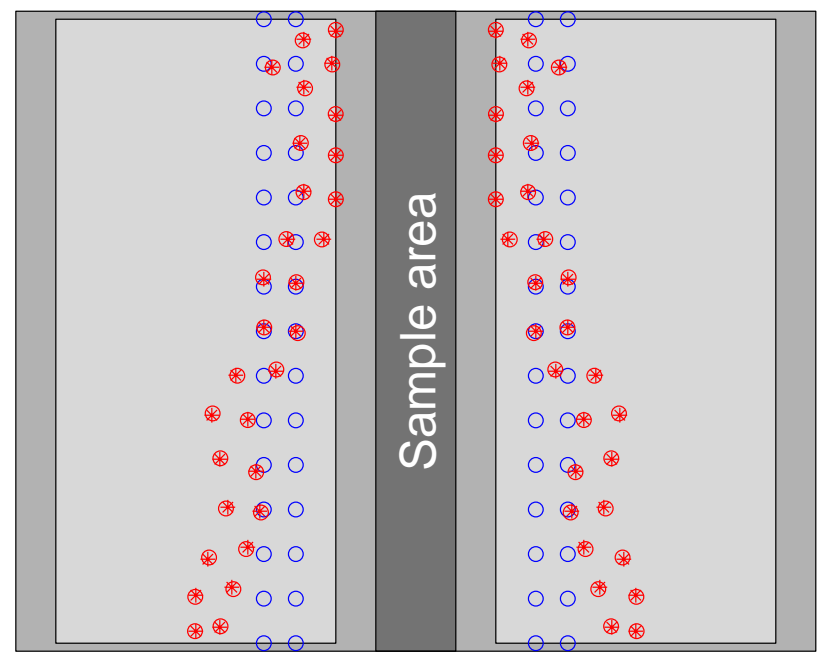

Initial pattern, $F\left(x_{m}, z_{m}, f\right)=1.41$

Optmized pattern, $F\left(x_{m}, z_{m}, f\right)=0.76$

* Optimized pattern (rounded), $F\left(x_{m}, z_{m}, f\right)=0.78$

Figure 10: Microphone locations. Blue circle - original locations. Red circle - optimized locations. Red asterisk - rounded optimized locations.

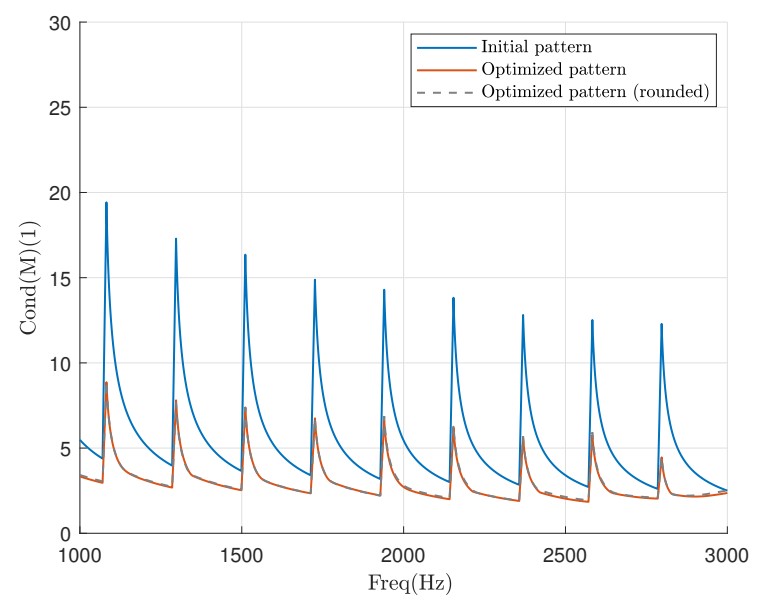

Figure 11: Condition number as a function of frequency for: initial, optimized and optimized with rounding pattern

To check that condition number of the optimized pattern is not sensitive to a minor change to $x$ or $z$, which could be induced by manufacturing and the finite size of 
the microphones, a sensitivity study is performed, where uniformly distributed noise with a maximum amplitude of $5 \mathrm{~mm}$ is added to $x$ and $z$ values at each microphone location. The unweighted linear average of condition number is evaluated for 10,000 independent runs with added noise. An average condition number of 2.95 and standard deviation of 0.07 are obtained. The distribution is shown in figure 12 As a reference, the linear average for the case without noise is 2.90 . Thus, one may conclude that the unavoidable uncertainties would not affect the performance of the chosen microphone pattern excessively.

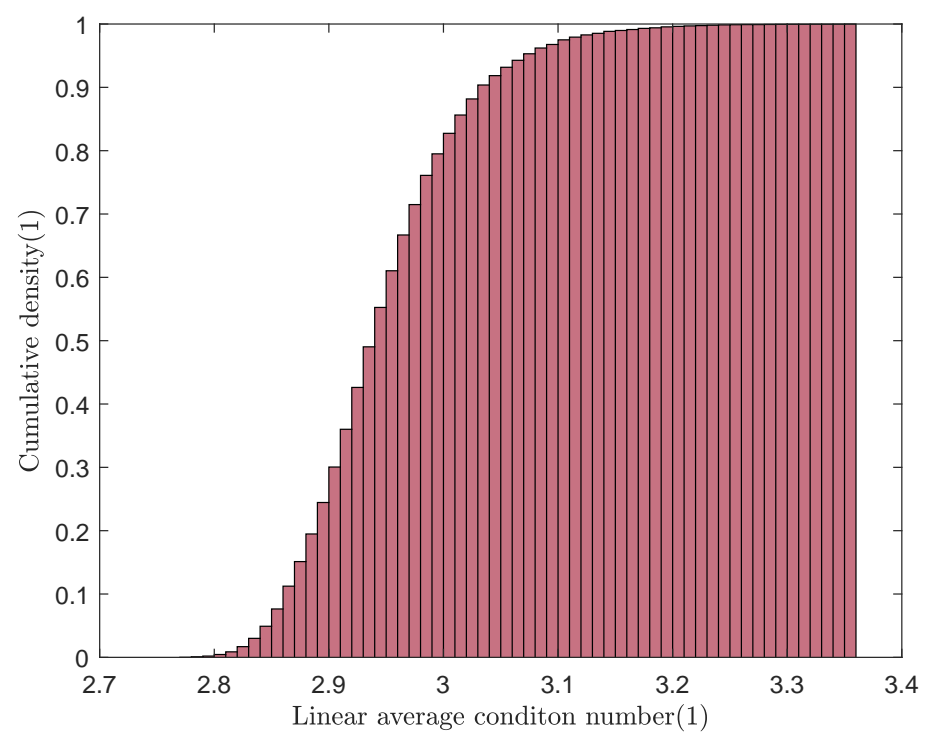

Figure 12: The distribution of runs over condition number

It is worth noting that several locations from the optimized pattern are in the close proximity of the boundaries, which means conducting the optimization with the two lines in the middle of the duct segment should even further improve the result. However, as mentioned earlier, the goal is to have a low condition number, that the current optimized one already serves the need.

It can be concluded that in absence of measurement errors, all microphone patterns will provide similar performance provided that the corresponding modal matrix is invertible. However, as in real-life experiments the measurement errors are unavoidable, 
the optimization is important to make the matrix inversion as robust as possible.

As a random pattern is likely to result in unwanted singularities other than at cut-on frequencies, the original pattern is not randomly chosen but based on the shape of the modes in a 2D waveguide (from which the number of microphones on a line is determined) and the shortest axial wavelength in the frequency range of interest (which is used to choose the spacing between the two lines), which yields only a singular modal matrix at the cut-on frequencies. This ensures a good starting point is reached before the optimization.

Although the chosen starting layout is usable for a broad frequency range, it is sub-optimal since it yields high condition number when one of the modes has a very long axial wavelength. For example, at frequencies slightly above a cut-on frequency, very high condition number can be observe, as shown in Figure 5. As a result, the original layout will result in a larger uncertainty on the results at these frequencies. For the optimized pattern, as shown in Figure 11, this issue has been greatly improved.

Besides, the optimization can tailor the microphone pattern to a specific measuring range, e.g., a combination of 500-1000 Hz and 1500-2000 Hz, whereas it is hard for the original pattern to achieve the same level of performance.

\subsection{Numerical models}

This part describes the setups of two numerical models: an idealized model for benchmark purpose and a realistic model for validation based on the model decomposition method.

\subsubsection{Idealized numerical model for bench marking}

The idealized numerical model is used to predict the absorption coefficient of a sample under oblique plane wave incidence in an idealized manner as described in section 2.2.3 based on the parameters retrieved from the impedance tube measurement. It can then serve as a benchmark for later sections. Figure 13 shows a schematic representation of the idealized numerical model. COMSOL Multiphysics ${ }^{\circledR} 5.4$ is used with the pressure acoustics module which solves the Helmholtz equation in the frequency domain. Bloch-Floquet periodic boundary conditions are applied to the 
boundaries perpendicular to the air sample interface. A perfectly matched layer (PML) is added to both ends of the acoustic domain to remove unwanted reflections. The foam is modeled as an equivalent fluid with parameters retrieved experimentally using the method described in section 4.1. A plane wave incidence is added to the region to the left of the sample (white region to the left of the sample area) as a background pressure field. The reflection coefficient and transmission coefficient from the simulation are approximated as:

$$
\begin{gathered}
R=\frac{\overline{P_{s, l_{1}}}}{\overline{P_{i, l_{1}}}} \\
T=\frac{\overline{P_{t, l_{2}}}}{\overline{P_{i, l_{1}}}}, \\
\alpha=1-|R|^{2}-|T|^{2},
\end{gathered}
$$

where $l_{1}$ and $l_{2}$ are two lines that are $8 \mathrm{~cm}$ away from the surface of the sample (figure $13, \overline{P_{s, l_{1}}}$ is the average of scattered field pressure over $l_{1}, \overline{P_{i, l_{1}}}$ is the average of incidence field pressure over $l_{1}$ and $\overline{P_{t, l_{2}}}$ is the average of transmitted field pressure over $l_{2}$, as in the acoustic far field, evanescent components are negligible and the acoustic pressure field can be described by only the propagating oblique plane waves of interest. A mixture of triangular and quad meshes are used. The maximum element size is set to be $\frac{1}{10}$ of the minimum acoustic wavelength considered. The frequency range for each simulation is from $1000 \mathrm{~Hz}$ to $3000 \mathrm{~Hz}$ with a step of $20 \mathrm{~Hz}$.

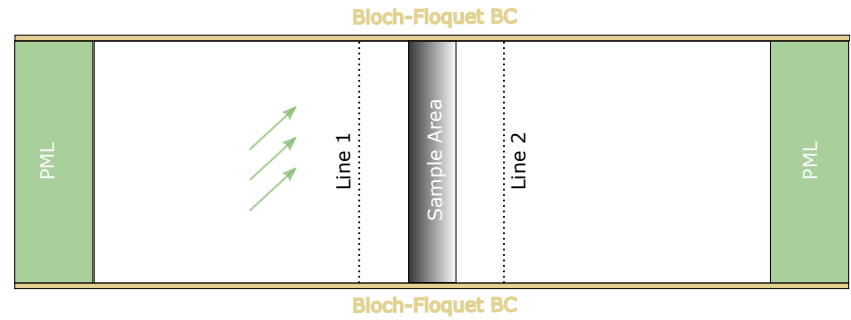

Figure 13: Numerical model for the idealized simulation

\subsubsection{Numerical model for modal characterization}

To check that the modal decomposition method with optimized microphone locations holds, a virtual experiment is performed in COMSOL Multiphysics ${ }^{\circledR} 5.4$ with a 
waveguide of the dimensions described in section $4.2(0.8 \mathrm{~m}$ by $0.045 \mathrm{~m}$ by $1 \mathrm{~m})$. The Pressure Acoustics module is used. An illustration of the numerical model is shown in figure 14

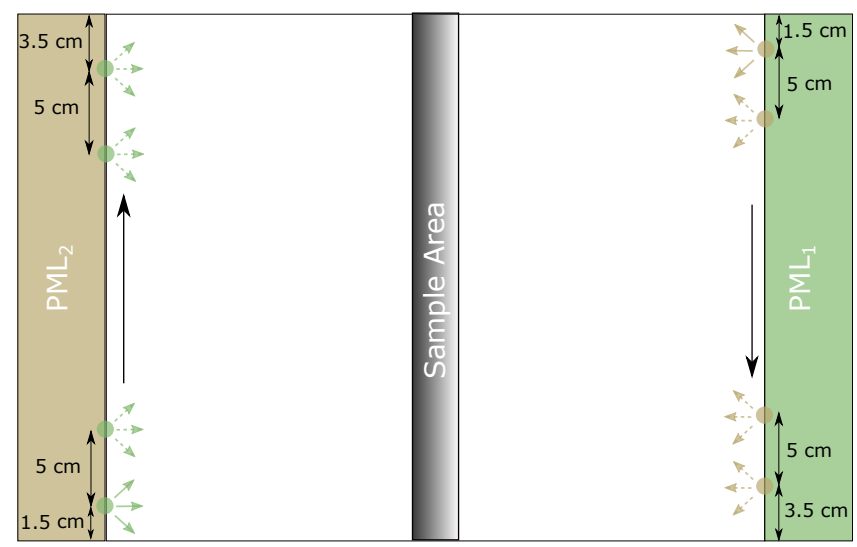

Figure 14: Numerical model for the virtual experiment

In the simulation, the sample of $0.8 \mathrm{~m}$ width is placed in the middle of the $2 \mathrm{D}$ waveguide. To ensure a full rank of the modal matrix at the frequency range of interest, a monopole sound source is placed at 32 locations either at the left (yellow) or right (green) boundary of the waveguide based on the reasoning in section 2.2.2 as shown in figure 14 . It is worth noting that the source pattern at each opening is not symmetric in terms of the central axis line of the waveguide. The aim to create runs as independent as possible. Sound hard boundaries (rigid walls) are added to both sides of the waveguide. A PML is appended to the opposite side of the sample with regard to the sound source to enable an anechoic termination. The maximum element size is set to be $\frac{1}{10}$ of the minimum wavelength considered. For each simulation, the acoustic pressure at locations obtained in section 4.2 is extracted. The frequency range for each simulation is again from $1000 \mathrm{~Hz}$ to $3000 \mathrm{~Hz}$ with a step of $20 \mathrm{~Hz}$.

\subsection{Experimental setup for modal characterization}

As shown in figure 15, two $1 \mathrm{~cm}$ thick plexi-glass plates and two $0.5 \mathrm{~cm}$ thick plexiglass stripes are used to form the waveguide. The internal dimensions of the waveguide 
are $0.8 \mathrm{~m}$ (left to right in the figure) by $1 \mathrm{~m}$ (top to bottom) by $0.045 \mathrm{~m}$ (height), which being the same as the ones used in the numerical setup.

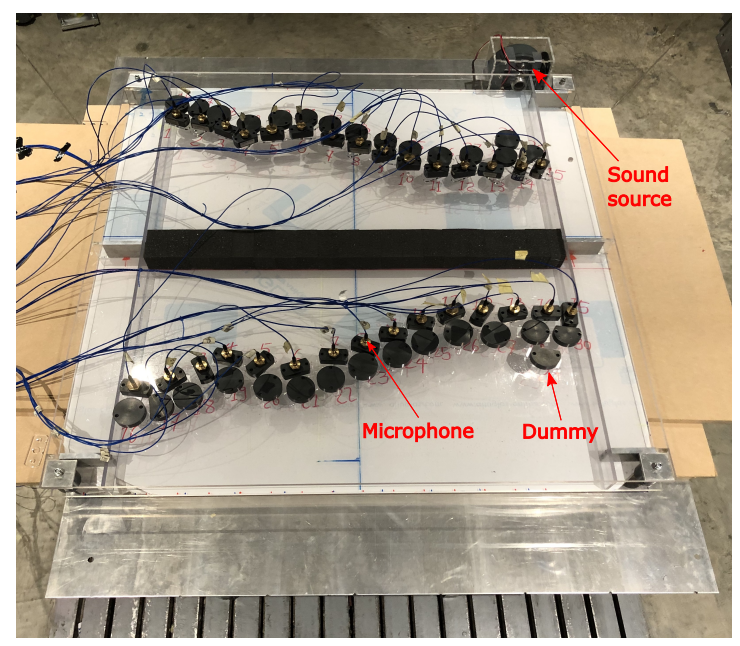

Figure 15: The experimental setup

A horn driver (Monacor KU-516, figure 16) is chosen as the sound source. Thirtytwo separate loudspeaker locations (same as those in the simulation) are chosen for the measurements. On each side, sixteen loudspeaker locations with a spacing of $5 \mathrm{~cm}$ are chosen (which are the same as those from the simulation). A total number of 60 holes for the microphones are drilled using a $\mathrm{CNC}$ router at the locations obtained from the optimization, as described in section 2.3. A total number of 30 1/4" PCB pressure field microphones (type 378C10) are flush-mounted on the top plate to measure the acoustic pressures at given locations. It is worth noting that given the limited number of available microphones as compared to the number of microphone locations (30 as compared to 60), at each loudspeaker location two subsets of measurements are performed to cover all microphone locations which are then merged into one complete measurement in the post-processing. At each subset of the two measurements, the locations not occupied by a microphone are sealed by a dummy as shown in figure 15 . The acoustic pressure and frequency response functions (FRFs) are measured using Siemens Simcenter Testlab with two tethered Simcenter SCADAS Mobiles to provide enough input channels. A white noise signal is input to the sound source. The bandwidth is $8192 \mathrm{~Hz}$. To 
ensure accuracy, 100 runs are taken and averaged for each configuration with a hanning window with an overlap of $50 \%$. The microphones are calibrated by phase before the measurement.

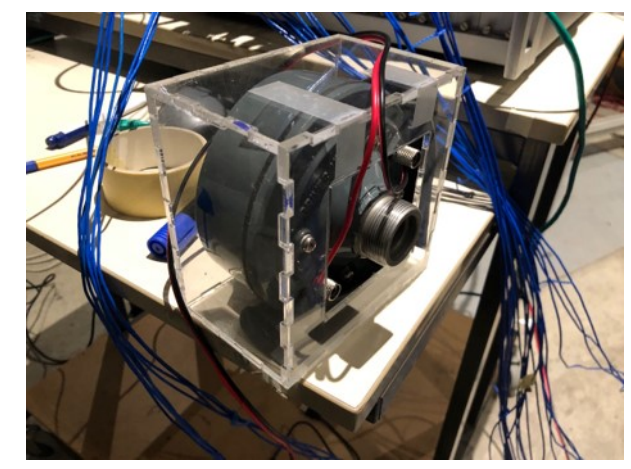

Figure 16: The horn driver used as the sound source in the experiment

\section{Results and discussions}

This section introduces the results from the material characterization study, numerical and the experimental studies on modal decomposition method. A good match is observed in both bases. Finally, the results are discussed.

\subsection{Sample characterization in a conventional impedance tube}

Figure 17] shows the equivalent fluid parameters of the sample retrieved from the impedance measurement using the two cavity method, which is in line with the curve trend of typical parameters for foams of this kind [21]. The spikes for the impedance curves around $1100 \mathrm{~Hz}$ is due to the impedance tube as they also appear in measurements for other type of samples using the same impedance tube. Figure 18 shows the absorption coefficient directly measured for the no-cavity case and that calculated based on equation 25), using the material parameters obtained with the two cavity method. As can be observed, the two absorption coefficient curves agree well, validating the correct retrieval of the material properties. 

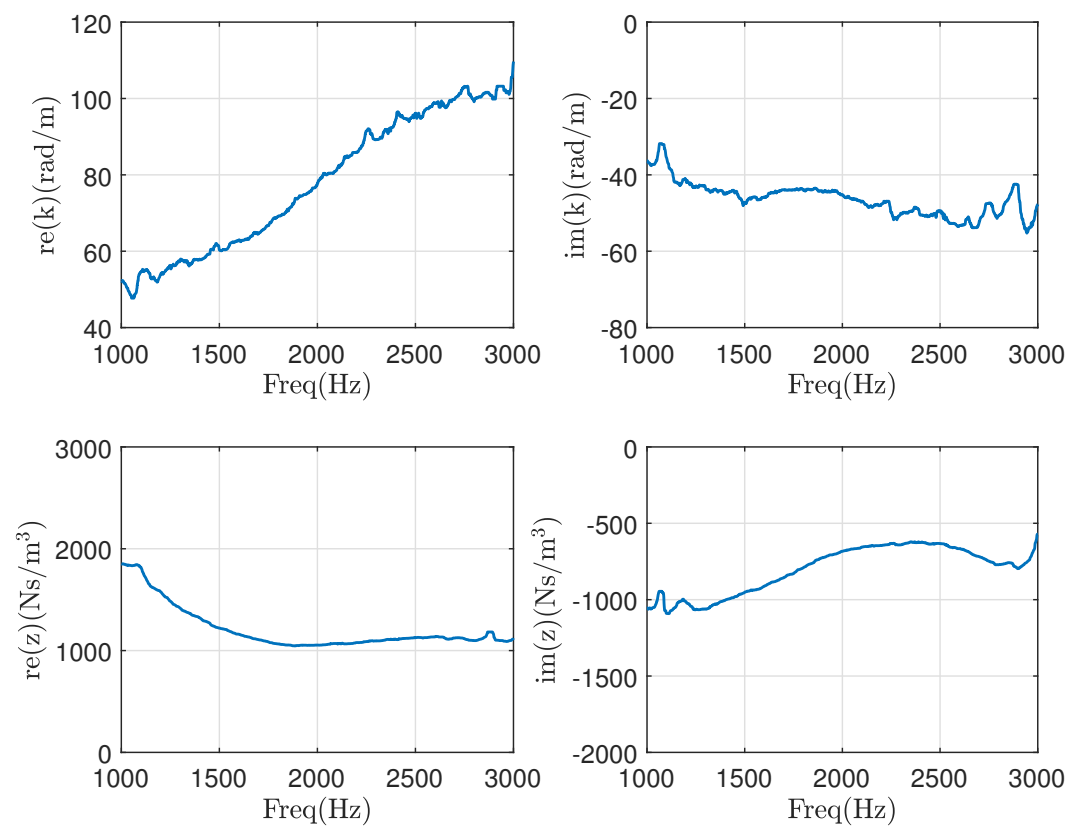

Figure 17: Retrieved equivalent wavenumber and impedance of the foam samples

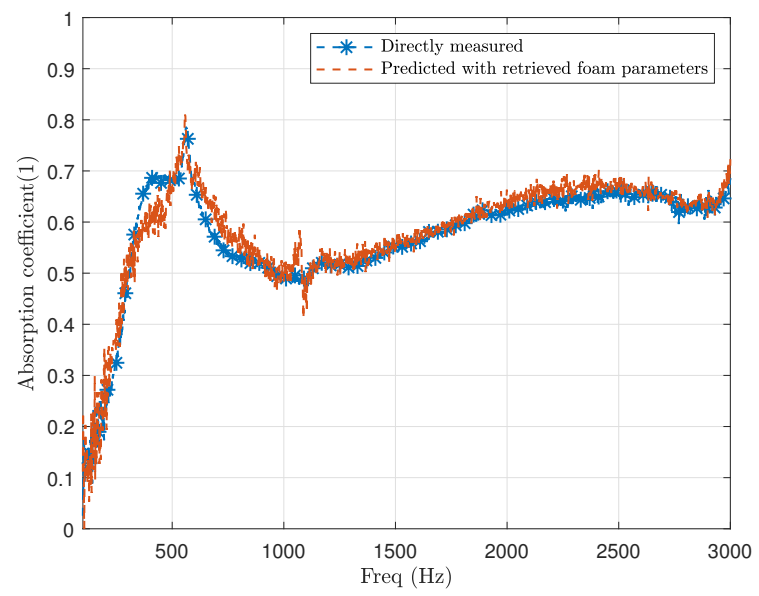

Figure 18: Absorption coefficient as a function of frequency for the sample with a rigid backing without a backing cavity 


\subsection{Numerical validation of the modal characterization}

Figure 19 shows the numerically retrieved absorption coefficient under normal incidence as a function of frequency and the angle-dependent absorption coefficient at $2000 \mathrm{~Hz}$, where a good agreement can be observed. It is worth pointing out that the peaks and dips of the $\alpha$ curve under normal incidence using the modal decomposition method are at the cut-on frequency of the different modes, where the modal matrix becomes singular.
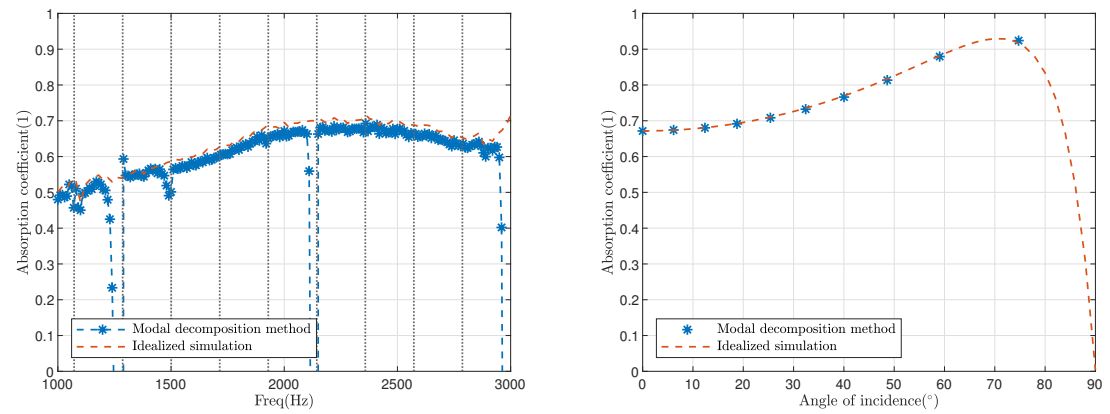

Figure 19: Absorption coefficient from simulation. Left - normal incidence $\alpha$ over frequency. Right - oblique incidence $\alpha$ at $2000 \mathrm{~Hz}$.

To mimic real-life measurements, additive white Gaussian noise of various SNRs are generated and added to each signal from the simulation independently. Three levels of SNR are used: 10, 20 and 30. As can be seen from figure 20 as the SNR increases, the absorption coefficient of both curves become less nervous and a better match can be observed with the prediction.

It should be noted that the study on the influence of SNR on the absorption coefficient does not compromise our effort on microphone location optimization, since the optimization process addresses the sensitivity of the modal matrix which is an inherent property of the system, whereas the SNR study emphasizes on the excitations that are applied to the system which does not rely on the system. It is safe to say that the final uncertainty level of the absorption coefficient curve is dependent on both system and excitation information.

To conclude, the numerical validation shows that the current methodology allows 

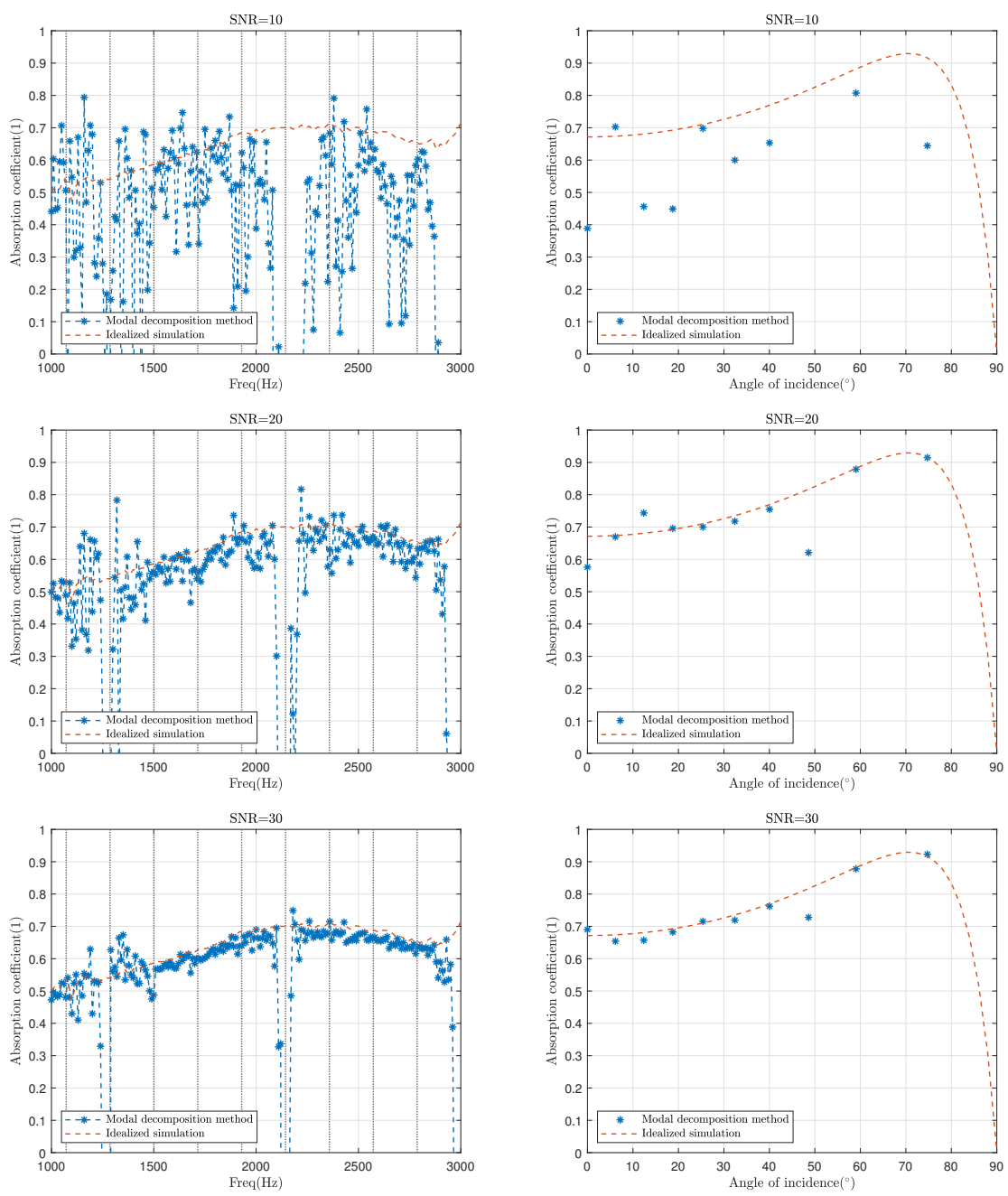

Figure 20: Absorption coefficient from simulation with noise of different level of SNR added. Left column normal incidence $\alpha$ over frequency. Right column - oblique incidence $\alpha$ at $2000 \mathrm{~Hz}$.

to obtain the angle-dependent $\alpha$.

\subsection{Experimental validation of the modal characterization}

Figure 21 shows the experimentally obtained absorption coefficient as a function of frequency under normal incidence and the angle-dependent absorption coefficient at $2000 \mathrm{~Hz}$. In general, a good match is observed for both cases between the prediction and the experiment for the sample between $1000 \mathrm{~Hz}$ and $3000 \mathrm{~Hz}$. 

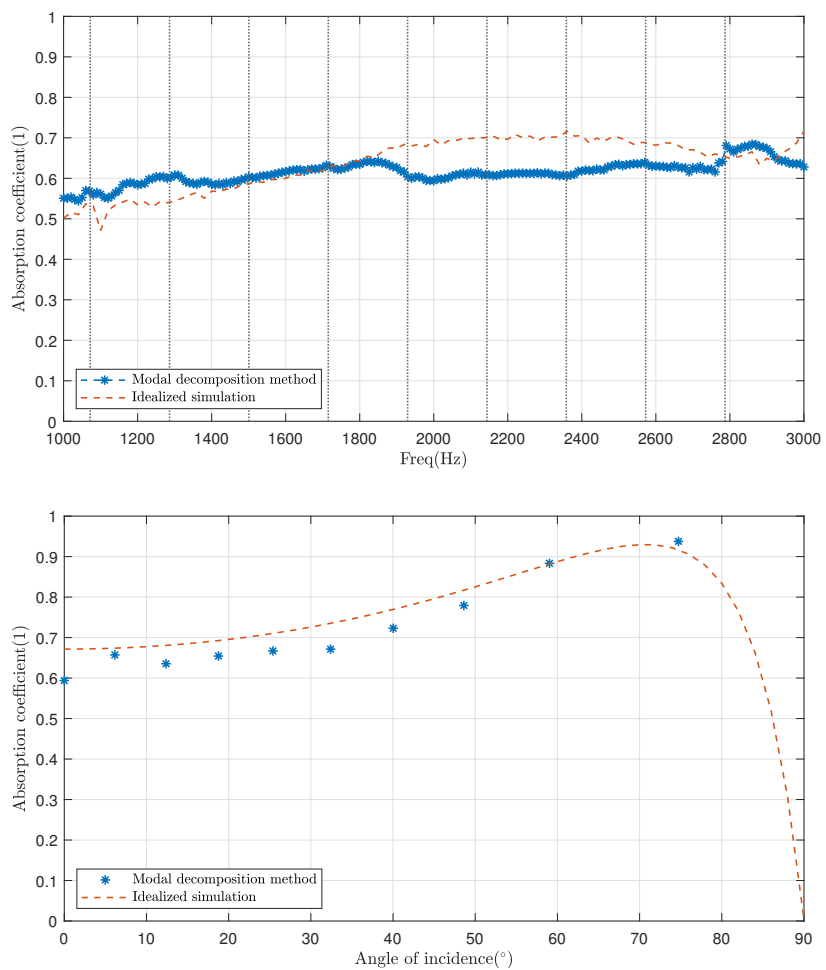

Figure 21: Absorption coefficient from experiment. Top - normal incidence $\alpha$ over frequency. Bottom oblique incidence $\alpha$ at $2000 \mathrm{~Hz}$

As a further validation, the sample of the same type and thickness is measured in a 2-port impedance tube. The measured $R$ and $T$ from both the 2-port impedance tube and the 2D waveguide are shown in figure 22, where a good match can be observed.

To even further improve the match, two measures can be taken. Firstly, the condition number of the modal matrix can be lowered by adding more microphone locations and optimizing over all locations in the frequency range. Secondly, as the measurement also depends on the excitations, adding more independent sound source locations should also help further improve the match. An optimization of the sound source locations considering the condition number of a matrix which is used to calculate the scattering matrix based on simulations or analytical model would be possible too.

Another observation that can be made from figure 21 is that the absorption coefficient 


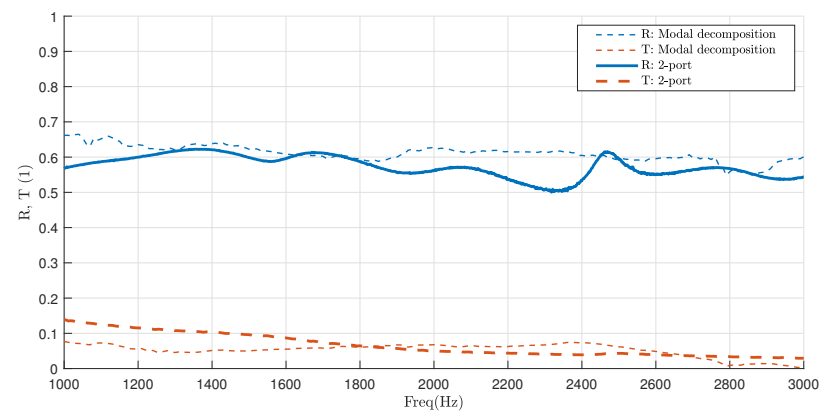

Figure 22: $R$ and $T$ measured from a 2-port impedance tube and the 2D waveguide

at $2000 \mathrm{~Hz}$ increases with angle of incidence then followed by a sudden drop. This can need to be larger

To conclude, the measured angle-dependent absorption coefficient agrees well with the prediction, validating the proposed method and workflow.

\section{Conclusions}

A novel method is reported to characterize the angle-dependent absorption coefficient of a sample using a modal decomposition method in a 2D waveguide with a rectangular cross-section. It is found that the locations of the microphones can greatly affect the condition number of the modal matrix thus the smoothness of the obtained 
absorption coefficient. Based on this reasoning, an optimization is performed to minimize the condition number of the modal matrix over the frequency range of interest. A sensitivity study of the optimized microphone locations shows that the optimized microphone locations are robust enough to artificially added noise on the microphone locations which mimics manufacturing error. The numerically obtained absorption coefficient for the idealized case and for the case using the proposed method agree well. A waveguide is fabricated with the dimensions from the numerical model with the layout of microphones from the optimization. The experimentally obtained absorption coefficients agree well with their numerical counterparts, validating that the modal decomposition method can be used to obtain a fairly accurate angle-dependent absorption coefficient of a sample. Given its easy and fast implementation and the

${ }_{430}$ sample-independent nature, this method holds high potential to be used at an early phase of product development or as a validation method for the research.

\section{Acknowledgments}

The research of Z. Zhang is funded by an Early Stage Researcher grant within the European Project PBNv2 Marie Curie Initial Training Network (GA 721615). The research of E. Deckers is funded by a grant from the Research Foundation - Flanders (FWO). The Research Fund KU Leuven is gratefully acknowledged for its support.

\section{References}

[1] ISO, 10534-2: 2001, Acoustics-Determination of sound absorption coefficient and impedance in impedance tubes-Part 2 (2001) 10534-2.

440 [2] J. Allard, N. Atalla, Propagation of sound in porous media: modelling sound absorbing materials 2e, John Wiley \& Sons, 2009.

[3] X. Ao, C. Chan, Far-field image magnification for acoustic waves using anisotropic acoustic metamaterials, Physical Review E 77 (2) (2008) 025601.

[4] E. Mommertz, Angle-dependent in-situ measurements of reflection coefficients using a subtraction technique, Applied Acoustics 46 (3) (1995) 251-263. 
[5] C. Nocke, In-situ acoustic impedance measurement using a free-field transfer function method, Applied Acoustics 59 (3) (2000) 253-264.

[6] R. Lanoye, G. Vermeir, W. Lauriks, R. Kruse, V. Mellert, Measuring the free field acoustic impedance and absorption coefficient of sound absorbing materials with a combined particle velocity-pressure sensor, The Journal of the Acoustical Society of America 119 (5) (2006) 2826-2831.

[7] M. Müller-Trapet, M. Vorländer, In-situ measurements of surface reflection properties, Building Acoustics 21 (2) (2014) 167-174.

[8] M. Tamura, J. F. Allard, D. Lafarge, Spatial fourier-transform method for measuring reflection coefficients at oblique incidence. ii. experimental results, The Journal of the Acoustical Society of America 97 (4) (1995) 2255-2262.

[9] J. Prisutova, K. Horoshenkov, J.-P. Groby, B. Brouard, A method to determine the acoustic reflection and absorption coefficients of porous media by using modal dispersion in a waveguide, The Journal of the Acoustical Society of America 136 (6) (2014) 2947-2958.

[10] S. Sack, M. Åbom, et al., On acoustic multi-port characterisation including higher order modes, Acta Acustica united with Acustica 102 (5) (2016) 834-850.

[11] S. Sack, M. Åbom, Investigation of orifice aeroacoustics by means of multi-port methods, Journal of sound and vibration 407 (2017) 32-45.

${ }_{465}$ [12] S. Sack, M. Shur, M. Åbom, M. Strelets, A. Travin, Numerical eduction of active multi-port data for in-duct obstructions, Journal of Sound and Vibration 411 (2017) 328-345.

[13] A. Sitel, J.-M. Ville, F. Foucart, Multiload procedure to measure the acoustic scattering matrix of a duct discontinuity for higher order mode propagation conditions, The Journal of the Acoustical Society of America 120 (5) (2006) 2478-2490.

[14] H. Denayer, Flow-acoustic characterization of duct components using multi-port techniques, Ph.D. thesis, KU Leuven (2017). 
[15] T. Schultz, L. N. Cattafesta III, M. Sheplak, Modal decomposition method for acoustic impedance testing in square ducts, The Journal of the Acoustical Society of America 120 (6) (2006) 3750-3758.

[16] A. D. Pierce, R. T. Beyer, Acoustics: An introduction to its physical principles and applications. 1989 edition (1990).

[17] J. Lavrentjev, M. Åbom, Characterization of fluid machines as acoustic multi-port sources, Journal of Sound and Vibration 197 (1) (1996) 1-16.

480 [18] I. The MathWorks, MATLAB Optimization Toolbox, Natick, Massachusetts, United State (2019).

URL https://nl.mathworks.com/products/optimization

[19] H. Utsuno, T. Tanaka, T. Fujikawa, A. Seybert, Transfer function method for measuring characteristic impedance and propagation constant of porous materials, The Journal of the Acoustical Society of America 86 (2) (1989) 637-643.

[20] R. Boonen, P. Sas, W. Desmet, W. Lauriks, G. Vermeir, Calibration of the two microphone transfer function method with hard wall impedance measurements at different reference sections, Mechanical Systems and Signal Processing 23 (5) (2009) 1662-1671.

490 [21] Y. Salissou, R. Panneton, Wideband characterization of the complex wave number and characteristic impedance of sound absorbers, The Journal of the Acoustical Society of America 128 (5) (2010) 2868-2876. 